\title{
Avaliação das Condições de Envelhecimento do Sistema Catalítico Versatato de Neodímio/hidreto de Di-isobutilalumínio/cloreto de t-butila na Polimerização de 1,3-Butadieno
}

\author{
Neusa Maria Tocchetto Pires, Bluma Guenther Soares \\ Instituto de Macromoléculas Professora Eloisa Mano, Universidade Federal do Rio de Janeiro \\ Fernanda Margarida Barbosa Coutinho ${ }^{\dagger}$, André Luiz Carneiro Simões, Marcos Antonio da Silva Costa \\ Instituto de Química, Universidade do Estado do Rio de Janeiro
}

Resumo: O objetivo deste trabalho foi estudar sistemas catalíticos ternários constituídos por versatato de neodímio, cloreto de t-butila e hidreto de di-isobutil-alumínio para a polimerização de 1,4-cis de butadieno em hexano. Foi investigado o efeito do envelhecimento natural do catalisador ( 1 a 60 dias a 8,5 ${ }^{\circ} \mathrm{C}$ ) sobre a conversão, a atividade catalítica e a micro- e macroestrutura do polibutadieno. Foi estudado também o efeito do envelhecimento forçado $\left(40^{\circ} \mathrm{C}\right)$, das diferentes fases do preparo do catalisador sobre as características da polimerização e do polímero sintetizado. Os polímeros foram caracterizados por cromatografia de exclusão por tamanho (SEC), espectroscopia na região do infravermelho (FT-IR), viscosimetria em solução e no estado fundido. O envelhecimento forçado das diferentes fases do catalisador (F1, F2, F3 e F4) afetou a conversão e a atividade catalítica de maneiras distintas. Os resultados de conversão e atividade catalítica mostraram que a fase mais afetada pelo envelhecimento forçado a $40^{\circ} \mathrm{C}$ foi a F1, etapa em que o versatato de neodímio é adicionado à solução de hidreto de di-isobutil-alumínio (Catalisador 447). A microestrutura não se alterou com o envelhecimento natural do catalisador enquanto que a macroestrutura mostrou dependência desta variável. Foi observado um ligeiro aumento na conversão e na atividade catalítica à medida que o tempo de envelhecimento do catalisador aumentou de 1 para 10 dias.

Palavras-chave: Catalisador Ziegler-Natta, neodímio, envelhecimento, polibutadieno.

\section{Evaluation of Aging Conditions of the Catalyst System Comprising Neodymium Versatate/ di-isobutylaluminium Hydride /t-butyl Chloride in the Polymerization of 1,3-Butadiene}

\begin{abstract}
This work studied ternary catalyst systems composed of neodymium versatate, t-butyl chloride and diisobutylaluminium hydride used in the cis-1,4 polymerization of butadiene in hexane. The effect of natural aging of the catalyst ( 1 to 60 days at $8.5^{\circ} \mathrm{C}$ ) on the conversion, catalyst activity and on the micro and macrostructure characteristics of polybutadiene was investigated. The effect of forced aging by heating $\left(40^{\circ} \mathrm{C}\right)$, of the different phases of the catalyst preparation on the polymerization characteristics and the synthesized polymer was also studied. The polymers were characterized by size exclusion chromatography (SEC), Fourier transform infrared spectroscopy (FT-IR) and viscosimetry in solution and melted-state. Accelerated aging of the different phases of the catalyst (F1, F2, F3 and F4) affected the conversion and the catalytic activity in distinct ways. The results of the conversion and catalytic activity showed that F1 was the most affected phase by accelerated aging at $40^{\circ} \mathrm{C}$. In this step the neodymium versatate was added to the solution of diisobutyl-aluminium hydride (Catalyst 447). The microstructure did not change with the natural aging of the catalyst while the macrostructure showed dependence on this variable. When the catalyst aging time increased from 1 to 10 days a slight increase in the conversion and the catalytic activity was observed.
\end{abstract}

Keywords: Ziegler-Natta catalyst, neodymium, aging, polybutadiene.

\section{Introdução}

Sistemas catalíticos baseados em neodímio têm atraído muita atenção para a polimerização de dienos conjugados $^{[1]}$. De todos os lantanídeos capazes de polimerizar dienos, neodímio é o que apresenta a mais alta atividade. A principal característica desses sistemas é que produzem polímeros com altíssimo teor de unidades cis a partir de vários dienos, como por exemplo, butadieno $^{[2]}$. A microestrutura dos polibutadienos, principalmente o teor de unidades cis, influencia significativamente as propriedades físicas do polímero puro e dos produtos vulcanizados, entretanto, estas não se modificam significativamente na faixa compreendida entre 25 e $80 \%$ de unidades cis, mas o fazem rapidamente após esses limites. Isso se deve ao fato de os polibutadienos possuírem a capacidade de cristalizar sob tensão, provocando a melhora das propriedades

Autor para correspondência: Marcos A. S. Costa, Instituto de Química, Universidade do Estado do Rio de Janeiro - UERJ, Rua São Francisco Xavier, 524, PHLC, CEP 20550-900, Rio de Janeiro, RJ, Brasil, e-mail: marcoscosta.iq.uerj@gmail.com †In memoriam. A Professora Fernanda M. B. Coutinho foi orientadora da Tese de Doutorado que deu origem aos resultados apresentados neste artigo. 
físicas do polímero. Esse efeito se acentua à medida que a pureza estérica do polímero aumenta. As principais propriedades do polibutadieno alto-cis vulcanizado são a alta elasticidade e resiliência, o baixo desenvolvimento de calor (heat build-up), a alta resistência à abrasão e ao crescimento do corte (cut-growth), a boa flexibilidade a baixas temperaturas e a alta resistência ao fendilhamento por fadiga. Aceita um teor mais elevado de negro de fumo e óleo, quando comparado com a borracha natural. Esse conjunto de caraterísticas faz do polibutadieno alto-cis um excelente elastômero para a indústria de pneumáticos ${ }^{[2-4]}$.

Em sua forma mais simples, os catalisadores de neodímio são obtidos a partir da reação entre compostos de neodímio e compostos de alquil-alumínio. Entretanto, catalisadores de interesse prático são obtidos somente se um doador de cloro estiver presente no sistema catalítico $^{[5]}$. Atualmente, o sistema catalítico à base de neodímio mais utilizado comercialmente é constituído por $\mathrm{Nd}$ (carboxilato)/haletos de alquilalumínio ou haletos orgânicos/alquilalumínio, preparados em um solvente do tipo hidrocarboneto ${ }^{[6]}$.

O modo de preparo do catalisador exerce grande influência sobre a natureza física, a atividade catalítica, a massa molar e a distribuição da massa molar do polímero. A microestrutura do polímero parece não ser afetada significativamente pelas mudanças do procedimento de síntese do sistema catalítico. Entretanto, a comparação dos dados existentes na literatura é dificultada devido à diversidade de condições empregadas pelos diferentes autores. Entre os vários procedimentos de preparo do sistema catalítico, citados na literatura, os mais relevantes referem-se à ordem de adição dos componentes; ao preparo do catalisador in situ, ou pré-formado; pré-formado por adição sequencial ou pré-mistura; envelhecimento do catalisador pré-formado em diferentes tempos e/ou temperaturas e preparo e/ou envelhecimento do catalisador na presença do monômero. A literatura relata, para os catalisadores à base de neodímio, a coexistência de diferentes sítios catalíticos, sensíveis à temperatura e ao tempo de envelhecimento, durante o preparo desses catalisadore $\mathrm{s}^{[2]}$. Porri ${ }^{[7]}$ estudou o sistema catalítico ternário $\mathrm{Nd}\left(\mathrm{C}_{7} \mathrm{H}_{15} \mathrm{COO}\right)_{3} / 3 \mathrm{AlEt}_{2} \mathrm{Cl} / 30 \mathrm{Al}$ (i$\mathrm{Bu})_{3}$, pré-formado, utilizando duas diferentes ordens de adição, $\mathrm{Nd}+\mathrm{Cl}+\mathrm{Al}$ (a) e $\mathrm{Nd}+\mathrm{Al}+\mathrm{Cl}$ (b), e diferentes tempos de contatos dos dois primeiros componentes antes da adição do composto clorado, para o procedimento (b). No primeiro caso a reação do composto de $\mathrm{Nd}$ com o composto clorado é rápida, ocorrendo a precipitação de $\mathrm{NdCl}_{3}$. Entretanto, a reação de alquilação do cloreto de neodímio pelo composto alquilalumínio é lenta e o catalisador gerado é heterogêneo. No segundo caso, a reação do composto de $\mathrm{Nd}$ com o composto alquilalumínio é lenta e não forma precipitado. O tempo de envelhecimento desta mistura, antes da adição do composto clorado, é o responsável pela homogeneidade ou heterogeneidade do catalisador. A adição do composto clorado 10 dias após o composto de $\mathrm{Nd}$ e o alquilalumínio serem misturados produziu catalisadores homogêneos ${ }^{[7]}$. Ricci $^{[8]}$ e Cabassi ${ }^{[9]}$ estudaram o sistema catalítico ternário $\mathrm{Nd}$ (octanoato) ${ }_{3} / \mathrm{AlEt}_{2} \mathrm{Cl} / \mathrm{Al}(\mathrm{i}-\mathrm{Bu})_{3}$, pré formado nesta ordem, envelhecido a $23^{\circ} \mathrm{C}, 0^{\circ} \mathrm{C}$ e $-18^{\circ} \mathrm{C}$ por até três meses. $\mathrm{O}$ composto de $\mathrm{Nd}$ e o de cloro foram misturados à temperatura ambiente. A mistura resultante foi levada à temperatura do envelhecimento e foi finalmente adicionado o composto de alquilalumínio. Os resultados obtidos indicaram uma atividade inicial maior para o catalisador envelhecido a temperatura ambiente. Entretanto, ao longo do período de envelhecimento o catalisador foi desativando enquanto que os catalisadores mantidos a baixas temperaturas ficaram mais ativos com o tempo de envelhecimento ${ }^{[9]}$. Oehme ${ }^{[10]}$ avaliou o efeito da ordem de adição dos componentes do sistema $\mathrm{Nd}$ (octanoato) ${ }_{3} / 1,5 \mathrm{AlEt}_{2} \mathrm{Cl} / 25 \mathrm{Al}(\mathrm{i}-\mathrm{Bu})_{3}, \quad$ pré-formado e envelhecido por 30 minutos, em duas diferentes concentrações de neodímio. Para a concentração mais baixa de catalisador as distribuições de massa molar foram aproximadamente monomodais, seguindo a ordem: $\mathrm{Al}+\mathrm{Nd}+\mathrm{Cl}>\mathrm{Al}+\mathrm{Cl}+\mathrm{Nd}>\mathrm{Nd}+\mathrm{Cl}+\mathrm{Al}$, enquanto que para a concentração de catalisador mais alta todas as distribuições foram bimodais, e a intensidade dessa bimobilidade seguiu a ordem: $\mathrm{Nd}+\mathrm{Cl}$ $+\mathrm{Al}>\mathrm{Al}+\mathrm{Nd}+\mathrm{Cl}>\mathrm{Al}+\mathrm{Cl}+\mathrm{Nd}$. Oehme ${ }^{[1]}$ avaliou também o efeito da ordem de adição dos componentes do sistema $\mathrm{Nd}$ (octanoato) ${ }_{3} / 1,5 \mathrm{AlEt}_{2} \mathrm{Cl} / 25 \mathrm{Al}(\mathrm{i}-\mathrm{Bu})_{3}$, em adição sequencial e envelhecido em diferentes tempos e temperaturas, obtendo distribuições de massa molar muito diferentes em função da variação destes parâmetros. A influência do envelhecimento do sistema ternário versatato de neodímio/hidreto de diisobutilalumínio/cloreto de t-butila vem sendo estudada por nosso grupo ${ }^{[12,13]}$. Foi verificado que as condições de envelhecimento não influenciaram a microestrutura do polímero. Catalisadores envelhecidos 10, 25, 40 e $60^{\circ} \mathrm{C}$ por 24 ou $48 \mathrm{~h}$ mostraram um aumento da massa molar do polímero com o aumento da temperatura de envelhecimento. A conversão da polimerização e a constante de velocidade de propagação praticamente não foram afetadas, quando o tempo de envelhecimento aumentou de 24 para $48 \mathrm{~h}^{[12]}$. Quando os catalisadores foram estudados nos tempos de $0,5,15,40,80$ e 160 dias e nas temperaturas de 10,25 e $40^{\circ} \mathrm{C}$, foi verificado que reações utilizando os catalisadores envelhecidos por 40 dias produziram polibutadieno com maior massa molar do que os demais catalisadores. Verificou-se também, uma tendência de maiores conversões das polimerizações com os catalisadores envelhecidos a $25^{\circ} \mathrm{C}^{[13]}$.

Neste trabalho, o objetivo foi estudar o efeito do envelhecimento natural do catalisador $\left(1\right.$ a 60 dias a $\left.8,5^{\circ} \mathrm{C}\right)$ sobre a conversão, atividade catalítica e características micro e macroestruturais do polibutadieno e também o efeito do envelhecimento forçado $\left(40^{\circ} \mathrm{C}\right)$, das diferentes fases de preparo do catalisador sobre as características da polimerização e do polímero sintetizado.

\section{Experimental}

\section{Materiais}

Para a síntese dos catalisadores foram utilizados hidreto de di-isobutilalumínio, DIBAH (Akzo-Nobel), versatato de neodímio (Rhodia) e cloreto de t-butila (Sigma-Aldrich) em solução em hexano seco. Para a polimerização foram utilizados 1,3-butadieno, $n$-hexano 
(Petroflex Indústria e Comércio), purificado e seco em peneira molecular de $3 \AA$ (Angstron), nitrogênio super-seco, (White Martins). O antioxidante, fosfito de trinonilfenila (TNPP) e os terminadores, Irganox 1076 e 3,5-di-t-butil-4- hidroxitolueno (BHT) foram utilizados na forma de soluções em hexano a 10 e $50 \%$, respectivamente.

\section{Síntese do catalisador}

O catalisador padrão foi preparado seguindo as seguintes etapas: uma solução de alquilalumínio foi introduzida, por meio de seringa, em um frasco de 300 $\mathrm{ml}$ vedado com septo de borracha. Em seguida, o frasco foi colocado em banho de água e gelo a $5^{\circ} \mathrm{C}$ e deixado por alguns minutos até que a temperatura atingisse o equilíbrio. Em seguida, uma solução de versatato de neodímio foi adicionada ao frasco, também por meio de seringa, e este foi agitado levemente para total mistura das duas soluções. Após 10 minutos, foi adicionado cloreto de tert-butila. A solução obtida foi mantida a $10^{\circ} \mathrm{C}$ durante, pelo menos, 10 horas antes de ser utilizada na polimerização. Para este estudo, foram preparados seis catalisadores segundo o procedimento de preparo do catalisador modelo, mantendo a razão molar $\mathrm{Al}: \mathrm{Nd}: \mathrm{Cl}$ constante e igual a 10,8:1:2,9. O preparo dos catalisadores foi dividido em quatro fases distintas:

- F1 - Adição do composto de neodímio sobre a solução do alquilalumínio;

- $\quad$ F2 - Adição do agente de cloração sobre a Fase 1;

- $\quad$ F3 - Envelhecimento do catalisador (F3);

- F4 - Estocagem do mesmo (F4)

As três primeiras fases do preparo do catalisador (F1, F2 e F3) foram submetidas, ao que se chamou de envelhecimento forçado, que consistiu em deixar a fase em estudo sob agitação durante $4 \mathbf{h}$ a $40^{\circ} \mathbf{C}$. A quarta fase (F4), foi igual para todos os catalisadores e consistiu em estocar o catalisador pronto, em repouso, durante $\mathbf{2}$ dias $\mathrm{a}-15^{\circ} \mathrm{C}$.

Dessa forma, foram preparados cinco catalisadores: um catalisador modelo, três catalisadores com uma das fases submetidas a envelhecimento forçado, e um catalisador tendo as três fases submetidas a envelhecimento forçado. As fases que não estavam em teste, seguiram o procedimento de preparo do catalisador modelo. A Tabela 1 mostra de maneira simplificada o procedimento adotado.

Com o objetivo de identificar diferenças de comportamento, essas fases do preparo dos catalisadores, foram submetidas a um "envelhecimento forçado", e a conversão e a atividade das reações, bem como a

Tabela 1. Condições do envelhecimento forçado das diferentes fases (F) dos catalisadores.

\begin{tabular}{lcccc}
\hline $\begin{array}{c}\text { Característica do } \\
\text { catalisador }\end{array}$ & $\mathbf{F 1}\left({ }^{\circ} \mathbf{C}\right)$ & $\mathbf{F 2}\left({ }^{\circ} \mathbf{C}\right)$ & $\mathbf{F 3}\left({ }^{\circ} \mathbf{C}\right)$ & $\mathbf{F 4}\left({ }^{\circ} \mathbf{C}\right)$ \\
\hline Modelo (448) & 8,5 & 8,5 & 8,5 & -15 \\
Teste de F3 (446) & 8,5 & 8,5 & 40 & -15 \\
Teste de F2 (449) & 8,5 & 40 & 8,5 & -15 \\
Teste de F1 (447) & 40 & 8,5 & 8,5 & -15 \\
Teste das 3 fases (445) & 40 & 40 & 40 & -15 \\
\hline
\end{tabular}

microestrutura, a viscosidade Mooney, a massa molar e a viscosidade em solução dos polímeros formados foram analisados.

Após o preparo, os catalisadores foram submetidos ao que se chamou de envelhecimento natural, que consistiu em mantê-los estocados, em repouso, na temperatura padrão, $8,5^{\circ} \mathrm{C}$, por um período que variou de 1 a 60 dias, antes de serem utilizados na polimerização.

\section{Sintese do polímero}

A síntese do polímero foi realizada em planta piloto de aço inox, em atmosfera inerte (nitrogênio) equipada com 2 reatores Parr de 1 e 2 galões de capacidade. A reação de polimerização foi executada em um reator de 2 galões, onde os componentes foram adicionados na seguinte ordem: solução hexânica a $35 \%$ em peso de butadieno (Blend B), parte do hexano, catalisador e o restante do hexano. O catalisador foi alimentado ao reator por intermédio de um cilindro de aço inox de $50 \mathrm{ml}$ de capacidade, acoplado ao reator. Esse cilindro foi carregado com o catalisador, sob fluxo de nitrogênio, por intermédio de seringa. $\mathrm{O}$ catalisador contido no cilindro de $50 \mathrm{ml}$ foi então adicionado ao reator, carreado pelo restante do solvente, após a massa reacional (Blend $\mathrm{B}$ mais solvente) atingir a temperatura definida para o início da reação. Todos os componentes foram adicionados ao reator por diferença de pressão. As reações foram conduzidas adiabaticamente, após a temperatura inicial de reação estabelecida ser alcançada, a camisa do reator foi drenada e as reações foram conduzidas sem controle de temperatura até que o pico de temperatura, decorrente do calor de polimerização, seja atingido. A partir desse ponto, a temperatura da reação foi novamente controlada, sendo mantida em $95^{\circ} \mathrm{C}$.

A polimerização foi acompanhada através da determinação do teor de sólidos durante todo o tempo estabelecido para a polimerização, e/ou após a reação ter atingido conversão total, ou ainda, após a estabilização da conversão. O cemento (polímero obtido pela reação de polimerização em solução) foi transferido para o segundo reator, de 1 galão de capacidade, inertizado, já contendo o terminador, solução de BHT em hexano, a $50 \%$ em peso. A reação de terminação do polímero foi conduzida, sob agitação, a $60^{\circ} \mathrm{C}$, por pelo menos 20 minutos. Em seguida, o cemento foi estabilizado, utilizando-se uma solução de TNPP e Irganox 1076 em hexano, a $10 \%$ em peso de TNPP, sendo utilizada uma razão TNPP/Irganox 1076 igual a 1:2. Após o término das etapas de terminação e estabilização do polímero, o cemento é descarregado para um recipiente para posterior coagulação. A coagulação foi realizada em um recipiente de aço inox, de 20 litros de capacidade, contendo água a $90^{\circ} \mathrm{C}$, mantida sob vigorosa agitação mecânica, por intermédio de um agitador pneumático com impelidor de alto cisalhamento. O cemento foi vertido lentamente no recipiente, sob intensa agitação.

Terminada a coagulação, os grumos de borracha foram recolhidos com uma peneira, picados manualmente, pois esse polímero apresenta uma tendência alta em se aglomerar formando uma massa única de polímero, e em seguida são colocados em uma bandeja de aço inox 
perfurada. A borracha coagulada foi levada à estufa para eliminação da água retida nos grumos durante o processo de coagulação. A secagem foi realizada, na própria bandeja de aço perfurada, em estufa com circulação forçada de ar, a $60-65^{\circ} \mathrm{C}$, durante pelo menos 12 horas.

\section{Caracterização dos polímeros}

A medida da massa molar (Mw e Mn) e distribuição da massa molar foi realizada por meio de cromatografia de exclusão por tamanho (SEC), em um sistema HPLC da Waters (Waters 600), equipado com detectetor de índice de refração; tetra-hidrofurano (THF) como solvente, com fluxo de $1 \mathrm{~mL} / \mathrm{min}$; análise realizada a $30^{\circ} \mathrm{C}$; utilizando um conjunto de 4 colunas de syragel de $5 \mu$ de diâmetro e porosidades equivalentes à faixa compreendida entre 5 x $10^{2}$ a $10^{5} \AA ̊$ e calibrado com padrões monodispersos de poliestireno. As soluções poliméricas foram preparadas a $0,1 \%$ em massa em THF.

A viscosidade Mooney, ML(1+4), foi determinada segundo o método ASTM D $1646^{[14]}$, a $100^{\circ} \mathrm{C}$, em um viscosímetro modelo MV2000 da Alpha Technology. A medida da viscosidade de uma borracha no viscosímetro de cisalhamento a disco (viscosidade Mooney) é indicada pelo torque necessário para girar o rotor envolvido por uma amostra de borracha e fechado na cavidade de um molde, sob condições específicas. A amostra é condicionada à temperatura ambiente $\left(23 \pm 3^{\circ} \mathrm{C}\right)$ e posteriormente laminada para expelir o ar da amostra. Durante a análise a amostra é pré-aquecida a $100^{\circ} \mathrm{C}$, durante 1 minuto e a medição da viscosidade Mooney é feita em 4 minutos.

A microestrutura dos polibutadienos foi determinada em espectrômetro de infravermelho com transformada de Fourier (FTIR), Spectrum One - Perkin-Elmer, na forma de filmes vazados sobre células de $\mathrm{KBr}$, preparados a partir de soluções a $2 \%(\mathrm{p} / \mathrm{v})$ em clorofórmio. Os teores dos diferentes tipos de unidades repetitivas foram obtidos, segundo método descrito na literatura ${ }^{[15]}$, utilizando as absorbâncias a $725 \mathrm{~cm}^{-1}$ (1,4 cis), $910 \mathrm{~cm}^{-1}$ (1,2-vinila) e $965 \mathrm{~cm}^{-1}$ (1,4-trans).

Para fazer a integração de cada banda, foram traçadas linhas de base individuais, utilizando os seguintes valores para os limites de integração: de 542 a $895 \mathrm{~cm}^{-1}$ (cis1,4), de 898 a $925 \mathrm{~cm}^{-1}$ (1,2-vinila) e de 944 a $976 \mathrm{~cm}^{-1}$ (trans-1,4). O cálculo dos valores das absorbâncias foi feito utilizando o "Spectrum Software" v.3.01 da Perkin Elmer. O teor da cada tipo de unidade repetitiva foi obtido a partir das Equações 1 a 4, estabelecidas por Schröder ${ }^{[15]}$, considerando-se o total de insaturações igual a $100 \%$, uma vez que a espessura do filme polimérico não é conhecida.

$$
\begin{aligned}
& \% 1,4-\text { cis }=\left(A_{1} / 0,175\right) \times(100 / K) \\
& \% 1,4-\text { trans }=\left(A_{2} / 0,647\right) \times(100 / K) \\
& \% 1,2-\text { vinila }=\left(A_{3}\right) \times(100 / K) \\
& K=\left(A_{1} / 0,175+A_{2} / 0,647+A_{3}\right)
\end{aligned}
$$

Onde A1, A2 e A3 são respectivamente as absorbâncias das bandas correspondentes às configurações 1,4-cis, 1,4-trans e 1,2-vinila, localizadas em torno de $725 \mathrm{~cm}^{-1}$,
$965 \mathrm{~cm}^{-1}$ e $910 \mathrm{~cm}^{-1}$. As constantes $\mathbf{0 , 1 7 5}, \mathbf{0 , 6 4 7} \mathrm{e}$ 1,00, são as absortividades, $\varepsilon$, das mesmas bandas, respectivamente, tiradas da literatura ${ }^{[15]}$.

\section{Resultados e Discussões}

A literatura aponta os benefícios de se trabalhar em baixas temperaturas de preparo dos catalisadores à base de lantanídeos, para a polimerização 1,4-cis do butadieno. No início dos estudos preliminares, foram preparados alguns catalisadores à temperatura ambiente e constatou-se a sua baixa estabilidade, quando preparados nessa temperatura. Procurou-se então trabalhar na temperatura mais próxima possível da ambiente, de modo a manter a integridade do catalisador e evitar onerosos sistemas de refrigeração em uma planta industrial. A temperatura escolhida para o preparo do catalisador padrão foi $\mathbf{8 , 5 ^ { \circ }} \mathbf{C}^{[2]}$. Também para o envelhecimento do catalisador, a literatura mostra benefícios, para os sistemas catalíticos à base de lantanídeos. O tempo de envelhecimento a ser estudado foi determinado a partir de observações feitas em outros trabalhos da literatura ${ }^{[2]}$. Os catalisadores eram guardados em geladeira, por longos tempos, após o término de sua utilização. Periodicamente eram observados quanto ao aspecto visual, e eram utilizados em reações padrão. $\mathrm{O}$ resultado dessas observações, durante os demais estudos definiu a faixa de tempo em que seria realizado o estudo de envelhecimento.

Baixas temperaturas de preparo e envelhecimento do catalisador, assim como o próprio envelhecimento, favorecem a atividade e influenciam significativamente a massa molar e sua distribuição. São observadas distribuições bimodais para baixos tempos e baixas temperaturas de envelhecimento do catalisador.

A microestrutura do polibutadieno praticamente independe do modo de preparação do catalisador. Isso é uma característica da polimerização de butadieno com catalisadores lantanídicos.

A Figura 1 mostra os dados de conversão das reações realizadas e da atividade catalítica desses catalisadores.

Analisando-se os dados de conversão e atividade catalítica obtidos, e considerando-se o catalisador 448 , como padrão, conclui-se que a fase mais afetada pelo envelhecimento forçado a $40^{\circ} \mathrm{C}$, foi a primeira (F1), etapa em que o versatato de neodímio é adicionado à solução do hidreto de diisobutil-alumínio e, na qual provavelmente ocorre a alquilação do composto de neodímio (catalisador 447).

Em segundo lugar, a fase mais afetada foi a segunda (F2), quando o agente de cloração é adicionado à mistura do composto de neodímio com a solução do alquilalumínio, ou seja, a fase em que provavelmente ocorre a cloração do versatato de neodímio pelo cloreto de t-butila, por meio da troca de grupos radicais isobutila por cloro (catalisador 449). O envelhecimento forçado do catalisador pronto $(\mathbf{F 3})$, mostrou uma perda de atividade bem menos significativa do que aquela apresentada para as fases F1 e F2. E, finalmente, o envelhecimento forçado das três primeiras fases do catalisador produziu resultados semelhantes aqueles encontrados, quando somente a F1 foi submetida ao envelhecimento forçado, sugerindo que 


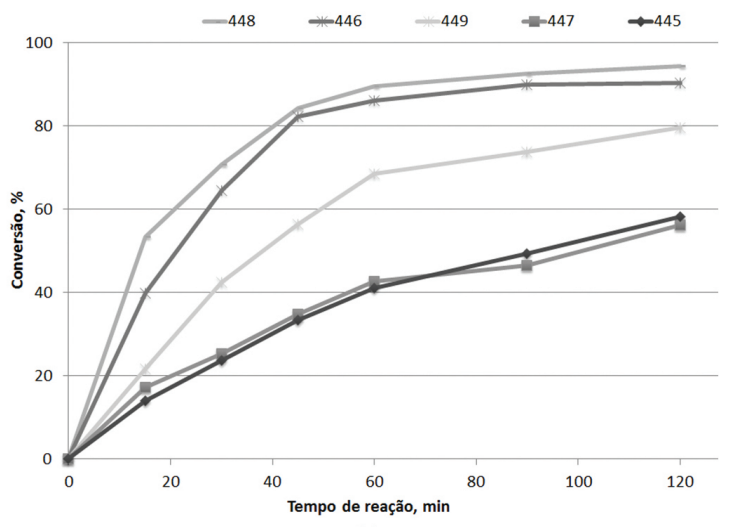

(a)

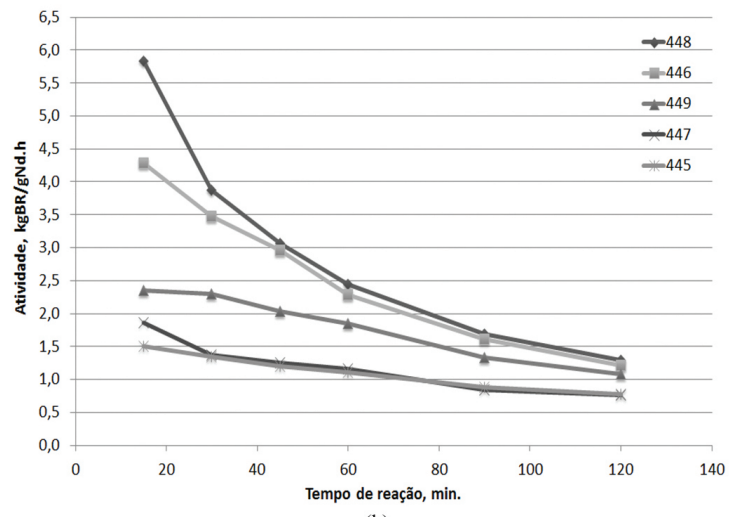

(b)

Figura 1. Influência do envelhecimento forçado das fases do preparo do catalisador sobre a conversão (a) e sobre a atividade catalítica (b). $[\mathrm{Nd}]=0,26 \mathrm{mmol} / 100 \mathrm{gBd} ;[\mathrm{Bd}]=12,5 \%$ em massa; razão molar $\mathrm{Al}: \mathrm{Nd}: \mathrm{Cl}=10,5: 1: 2,8$; temperatura inicial $=75^{\circ} \mathrm{C}$; tempo de reação=120 minutos; "reações adiabáticas". Bd= butadieno.

esta é a fase mais crítica, ou mais suscetível aos efeitos da temperatura.

Assim, considerando a perda de atividade do composto formado, por exposição à temperatura, a seguinte ordem poderia ser sugerida:

- Fase de alquilação $>>$ fase de cloração $>>$ fase de envelhecimento do complexo pronto $>$ catalisador padrão.

O fato de a etapa da alquilação, F1, ter um efeito tão acentuado sobre a atividade final do sítio ativo, corrobora a teoria de que o cloro desloca os radicais orgânicos isobutila do composto de neodímio, resultando na cloração do mesmo. Os dados sugerem que quando esta etapa de alquilação é deficiente, o mesmo acontece com a etapa de cloração. Sugerem também que a alquilação do composto de neodímio não é uma reação favorecida pela temperatura.

O teor de unidades 1,4-cis aumentou ligeiramente à medida que o envelhecimento forçado foi se mostrando mais efetivo com relação à influência exercida sobre as fases de preparo do catalisador. A variação ocorre em uma faixa muito pequena, $97,95 \%$ a $98,80 \%$, mas a tendência é inquestionável ${ }^{[2]}$. Novamente, o aumento registrado pelo teor de unidades 1,4-cis foi compensado por um decréscimo de mesmo valor absoluto, pelo teor de unidades 1,4-trans, mantendo-se praticamente constante o teor de unidades 1,2-vinila. Estes resultados sugerem que os sítios mais estáveis sejam os mais estereoespecíficos, pois o catalisador que apresentou o teor de unidades cis mais elevado foi justamente aquele em que a fase mais afetada pela temperatura, a fase da alquilação, foi submetida ao envelhecimento forçado, e apresentou a mais baixa atividade catalítica.

A literatura ${ }^{[16-19]}$ relata que a baixa atividade dos catalisadores à base de versatato de neodímio se deve à multiplicidade de estruturas que ele apresenta, que consistem de estruturas oligoméricas, com ligantes polares tais como a água, hidróxidos e carboxilatos, que saturam a esfera de coordenação do neodímio. Alguns dos átomos de neodímio localizados no meio das estruturas oligoméricas permanecem menos reativos, e outros localizados na parte mais externa das estruturas se transformam em centros ativos. Os autores relataram ainda, que os ligantes polares também consomem o alquil-alumínio, resultando em um decréscimo no número de sítios formados.

O mecanismo de formação da espécie ativa, proposto por Kwag[ ${ }^{[16]}$, dá suporte às afirmações feitas neste estudo, com relação à dependência exercida pela etapa de alquilação sobre a de cloração, e explica porque os sítios mais estáveis são os mais estereoespecíficos. A configuração anti que dá origem às unidades cis, é mais favorecida cineticamente, devido a fatores estereoquímicos, do que a configuração sin que dá origem às unidades trans, mais favorecida termodinamicamente. Assim, os átomos de neodímio mais internos nas estruturas oligoméricas do versatato de neodímio, que conseguem ser alquilados e posteriormente clorados, se transformam em centros ativos, de mais difícil acesso, favorecendo a aproximação da configuração anti. Estes mesmos centros ativos serão, por outro lado, os mais protegidos, e conseqüentemente os mais difíceis de serem desativados.

Os dados da Figura 2 apresentam uma relação bastante interessante entre o comportamento da viscosidade Mooney, dos polímeros obtidos com os cinco catalisadores estudados, e as conversões alcançadas nas respectivas reações.

Os sistemas de polimerização, onde foram utilizados os catalisadores 448 (padrão) e 446 (envelhecido após o término do preparo), apresentaram pequena diferença em relação à conversão atingida na reação, sugerindo que o número de sítios ativos dos dois sistemas seria muito próximo. Por outro lado, a diferença entre as viscosidades Mooney dos polímeros finais é extremamente significativa, sugerindo que os sítios que permaneceram ativos após o envelhecimento forçado, seriam os sítios responsáveis pela formação de polímero de alta massa molar ("sítios de alta massa molar") e estariam presentes em maior número antes do envelhecimento, uma vez que o aumento da viscosidade Mooney do polímero foi muito grande, se comparado à perda de conversão da reação. Oehme ${ }^{[11]}$, relata a existência de "sítios de alta massa molar" 
resistente a tempos e temperaturas de envelhecimento mais altos, para esses sistemas catalíticos.

Quando os sistemas de polimerização, onde foram utilizados os catalisadores 446 (envelhecido após o término do preparo), e 449 (envelhecimento da fase de cloração), são comparados, observa-se uma significativa perda na conversão da reação (sugerindo um decréscimo no número de sítios ativos) e um valor semelhante para a viscosidade Mooney. Na verdade, deve-se levar em conta a diferença de conversão, o que significa que a viscosidade Mooney deveria estar mais alta se a reação tivesse sido levada à mesma conversão que a reação do catalisador 446, pois a mesma quantidade de monômero teria sido inserida nas cadeias poliméricas. Assim, o aumento da viscosidade Mooney estaria relacionado, principalmente, com o decréscimo do número de sítios ativos (Figura 2). Por outro lado, o valor mais alto de viscosidade em solução, obtido para o polímero produzido com o catalisador 449 , confirma que a massa molar resultante, neste caso, é ainda mais alta do que aquela gerada com o catalisador 446, sugerindo diferentes estabilidades mesmo entre os "sítios de alta massa molar".

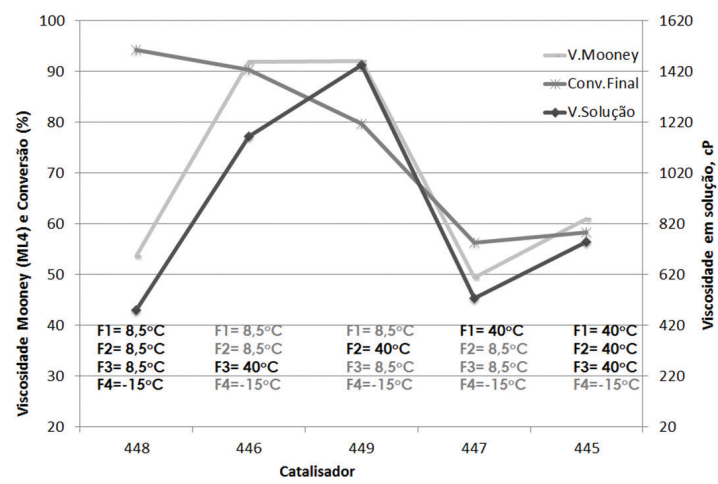

Figura 2. Efeito do envelhecimento forçado das fases do preparo do catalisador sobre a viscosidade Mooney, conversão e viscosidade em solução. $[\mathrm{Nd}]=0,26 \mathrm{mmol} / 100 \mathrm{gBd} ;[\mathrm{Bd}]=12,5 \%$ em massa; razão molar $\mathrm{Al}: \mathrm{Nd}: \mathrm{Cl}=10,5: 1: 2,8$; temperatura inicial $=75^{\circ} \mathrm{C}$; tempo de reação= 120 minutos; "reações adiabáticas". $\mathrm{Bd}=$ butadieno.

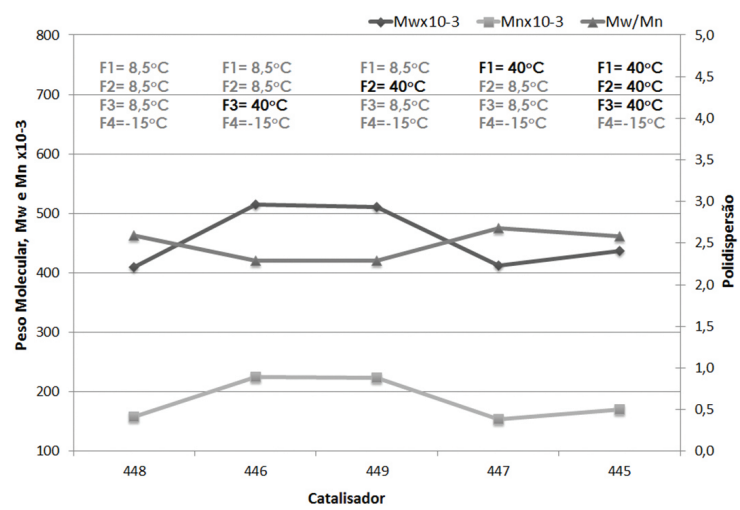

(a)
Conforme pode ser observado na Figura 3b, os polímeros produzidos com os catalisadores 446 e 449 apresentaram curvas de distribuição de massa molar, praticamente, idênticas, o que confirma a massa molar mais alta do polibutadieno produzido com o catalisador 449, uma vez que sua polimerização chegou a conversões mais baixas do que a polimerização realizada com o catalisador 446 . Considerando as estruturas oligoméricas do versatato de neodímio, sugeridas por Kwag ${ }^{[16]}$, poder-se-ia dizer que os átomos mais externos de neodímio podem ter sofrido uma desalquilação antes da substituição do radical alquila pelo cloro, e a localização diferenciada dos átomos de neodímio nessas estruturas, poderiam definir diferentes estabilidades para os centros ativos, ou diferentes características cinéticas.

Quando os sistemas de polimerização, onde foram utilizados os catalisadores 448 (padrão) e 447 (envelhecimento da fase de alquilação), são comparados, percebe-se que um número reduzido de centros ativos conseguiu sobreviver ao aquecimento, ou ainda que um número reduzido de átomos de neodímio conseguiu se manter alquilado, durante o aquecimento, dando origem a centros ativos, nas etapas subsequentes. A perda de conversão para o polímero produzido com o catalisador 447 foi muito alta, e a viscosidade Mooney significativamente mais baixa que o polímero de controle.

Finalmente, quando os catalisadores 447 (envelhecimento da fase de alquilação) e 445 (envelhecimento forçado das três primeiras fases do catalisador) foram comparados, observou-se que os resultados obtidos eram semelhantes, sugerindo que a fase da alquilação é a mais crítica, ou a mais suscetível aos efeitos da temperatura.

Como pode ser visto na Figura 3a, as massas molares, Mw e Mn, dos polímeros sintetizados, estão de acordo com os dados obtidos para a viscosidade Mooney e a viscosidade em solução, Figura 2.

\section{Efeito do tempo de envelhecimento natural do catalisador}

Neste estudo, seis catalisadores preparados de forma padrão, permaneceram estocados na temperatura padrão $\left(8,5^{\circ} \mathrm{C}\right)$ e foram mantidos em observação por um período

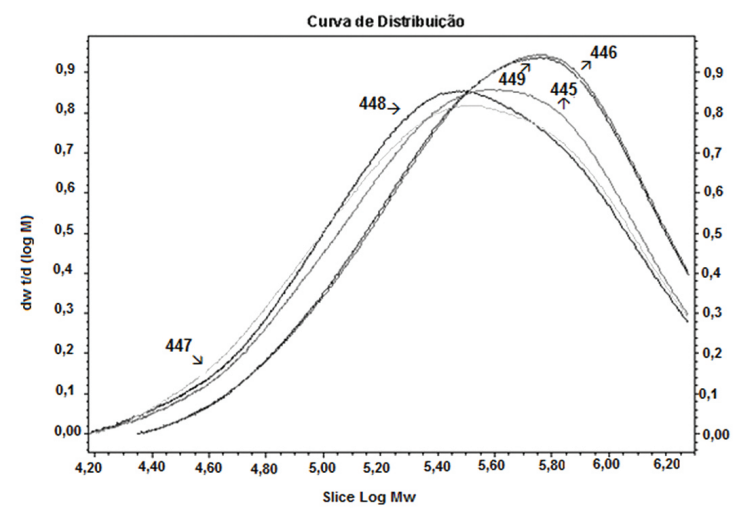

(b)

Figura 3. Influência do envelhecimento forçado das fases do preparo do catalisador sobre a massa molar numérica média $\left(\mathrm{M}_{\mathrm{n}}\right)$ e massa molar ponderal média $\left(\mathrm{M}_{\mathrm{w}}\right)$ (a) e sobre as curvas de distribuição da massa molar do polibutadieno (b). [Nd]=0,26mmol/100gBd; $[\mathrm{Bd}]=12,5 \%$ em massa; razão molar $\mathrm{Al}: \mathrm{Nd}: \mathrm{Cl}=10,5: 1: 2,8$; temperatura inicial $=75^{\circ} \mathrm{C}$; tempo de reação= 120 minutos; "reações adiabáticas". $\mathrm{Bd}=$ butadieno. 
de 1 a 60 dias, antes de serem utilizados na polimerização. Os polímeros produzidos foram analisados quanto à conversão, atividade catalítica, microestrutura, massa molar e distribuição de massa molar, viscosidade em solução e viscosidade Mooney. O envelhecimento foi chamado de natural, porque foi realizado nas condições padronizadas de estocagem dos catalisadores.

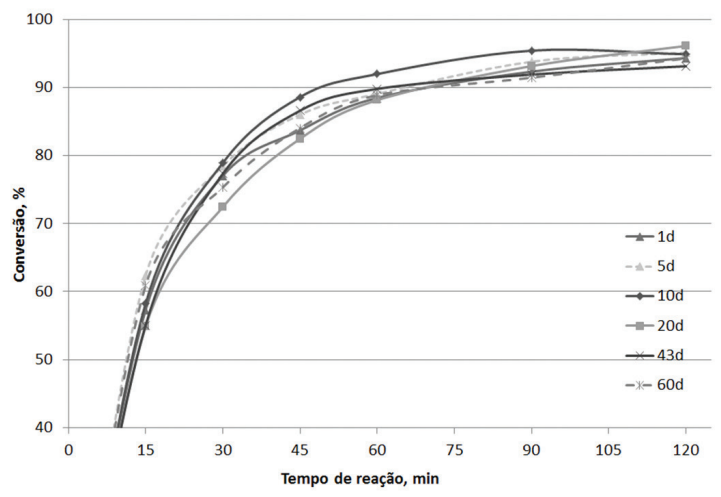

Figura 4. Efeito do envelhecimento natural do catalisador sobre a conversão da reação de polimerização. [ $\mathrm{Nd}]=0,26 \mathrm{mmol} / 100 \mathrm{gBd}$; $[\mathrm{Bd}]=12,5 \%$ em massa; razão molar $\mathrm{Al}: \mathrm{Nd}: \mathrm{Cl}=10,5: 1: 2,8$; temperatura inicial $=75^{\circ} \mathrm{C}$; tempo de reação $=120$ minutos; "reações adiabáticas"; envelhecimento do catalisador a $8,5^{\circ} \mathrm{C}$; $\mathrm{Bd}=$ butadieno

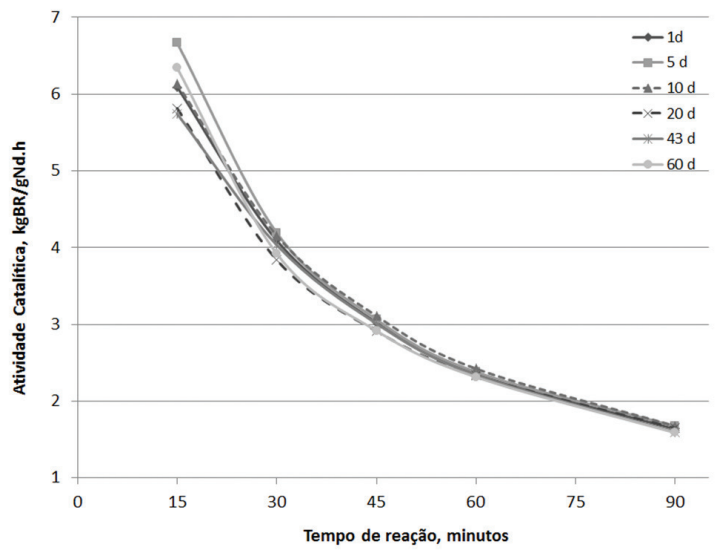

Figura 5. Influência do envelhecimento natural do catalisador sobre a atividade catalítica. $[\mathrm{Nd}]=0,26 \mathrm{mmol} / 100 \mathrm{gBd}$; $[\mathrm{Bd}]=12,5 \%$ em massa; razão molar $\mathrm{Al}: \mathrm{Nd}: \mathrm{Cl}=10,5: 1: 2,8$; temperatura inicial $=75^{\circ} \mathrm{C}$; tempo de reação= 120 minutos; "reações adiabáticas"; envelhecimento do catalisador a $8,5^{\circ} \mathrm{C}$; $\mathrm{Bd}=$ butadieno
Os resultados de conversão, ao longo da reação, obtidos para os seis diferentes tempos de envelhecimento, Figura 4, mostram um ligeiro aumento na conversão à medida que o tempo de envelhecimento do catalisador aumentou de 1 para 10 dias, parecendo haver um máximo discreto em 10 dias. Para tempos maiores de envelhecimento, embora a tendência não seja muito bem caracterizada, parece ocorrer uma ligeira desativação dos catalisadores com o aumento do tempo de envelhecimento percebido quando se analisa o conjunto como um todo.

Os dados de atividade catalítica, representados na Figura 5 mostram a mesma tendência. Os valores mais altos encontrados para a atividade catalítica em 15 minutos de reação, para os tempos de envelhecimento do catalisador de 5 e 60 dias, não invalidam o que foi dito anteriormente, pois aos 15 minutos de reação, a quantidade de monômero residual ainda é significativa, e a amostragem dos sólidos totais para a determinação da conversão pode ser prejudicada. A tendência à desativação, apresentada pelos catalisadores nas condições de envelhecimento utilizadas neste trabalho, parece estar de acordo com as conclusões relatadas por Cabassi ${ }^{[9]}$.

A massa molar, a viscosidade Mooney e a viscosidade em solução, obtidos para os polímeros envelhecidos, Tabela 2, aumentam com o tempo de envelhecimento até o $20^{\circ}$ dia, mantendo-se praticamente constantes para tempos maiores de envelhecimento. A polidispersão diminui significativamente, a partir do $10^{\circ}$ dia de envelhecimento, mantendo-se, constante a partir então. Estes dados sugerem uma maior transformação do sistema catalítico até o $20^{\circ}$ dia de envelhecimento, e a partir daí, o sistema tende a se estabilizar. A conversão final dos polímeros foi, praticamente, a mesma. A Figuras $6 a$, b mostra mais claramente a tendência acima descrita.

Este comportamento reforça a teoria da existência de vários tipos de sítios ativos com diferentes sensibilidades em relação ao tempo de envelhecimento ${ }^{[11]}$, e que os sítios mais instáveis seriam aqueles responsáveis pelos polímeros de baixa massa molar. Considerando-se o mecanismo de formação da espécie ativa proposto por $\mathrm{Kwag}^{[16]}$, onde esta é formada a partir de um composto de neodímio de estrutura oligomérica, poderia se dizer que os sítios mais instáveis seriam aqueles formados a partir dos átomos de neodímio mais externos à molécula, portanto mais suscetíveis à desativação, e consequentemente, responsáveis pelas massas molares mais baixas.

Tabela 2. Efeito do tempo de envelhecimento natural do catalisador sobre a massa molar e distribuição de massa molar, viscosidade Mooney e viscosidade em solução.

\begin{tabular}{|c|c|c|c|c|c|c|}
\hline Tempo (dias) & Conversão (\%) & $\operatorname{Mn} \times 10^{-3}(\mathrm{~g} / \mathrm{mol})$ & $\operatorname{Mw} \times 10^{-3}(\mathrm{~g} / \mathrm{mol})$ & Mw/Mn & V. Mooney $\left(\mathrm{ML}_{4}\right)$ & V. Solução (cP) \\
\hline 1 & 94,3 & 125 & 426 & 3,4 & 49,0 & 394 \\
\hline 5 & 95,1 & 117 & 418 & 3,6 & 56,1 & 522 \\
\hline 10 & 94,9 & 154 & 429 & 2,8 & 61,0 & 705 \\
\hline 20 & 96,1 & 166 & 446 & 2,7 & 66,2 & 741 \\
\hline 43 & 93,1 & 153 & 447 & 2,9 & 65,2 & 614 \\
\hline 60 & 94,3 & 158 & 448 & 2,8 & 67,3 & 742 \\
\hline
\end{tabular}

Temperatura de envelhecimento natural do catalisador $=8,5^{\circ} \mathrm{C}$. 


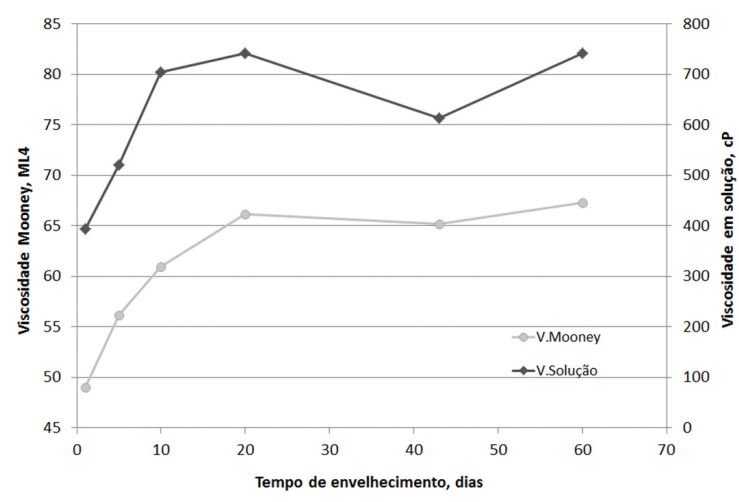

(a)

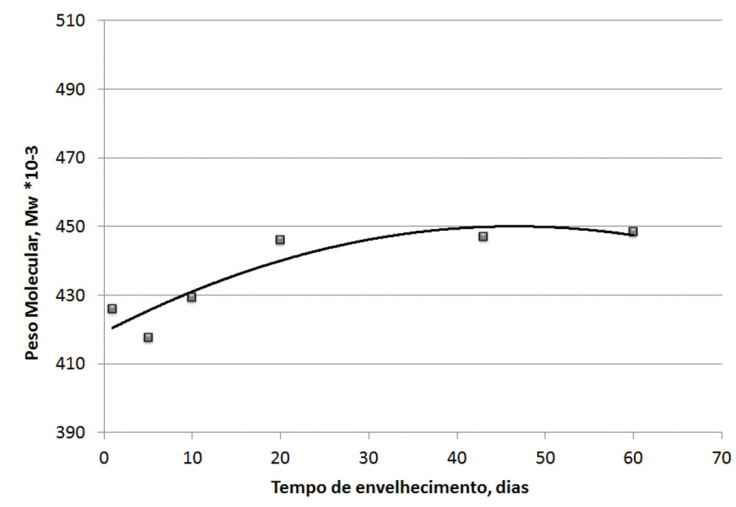

(b)

Figura 6. Influência do tempo de envelhecimento natural do catalisador sobre as viscosidades Mooney e em solução (a) e sobre a massa molar ponderal média do polibutadieno.

Tabela 3. Efeito do tempo de envelhecimento natural do catalisador sobre a microestrutura do polibutadieno.

\begin{tabular}{cccc}
\hline Tempo (dias) & 1,4-cis $(\%)$ & 1,4-trans $(\%)$ & 1,2-vinila (\%) \\
\hline 1 & 97,88 & 1,57 & 0,55 \\
5 & 97,94 & 1,50 & 0,55 \\
10 & 98,05 & 1,32 & 0,63 \\
20 & 97,69 & 1,60 & 0,71 \\
43 & 97,99 & 1,33 & 0,68 \\
60 & 98,03 & 1,29 & 0,69 \\
\hline
\end{tabular}

Temperatura de envelhecimento natural do catalisador $=8,5^{\circ} \mathrm{C}$.

A microestrutura dos polímeros formados não apresentou variação decorrente do envelhecimento dos catalisadores, nas condições utilizadas neste trabalho, conforme mostra a Tabela 3 .

Assim, para as condições de envelhecimento apresentadas nesse trabalho, e considerando que $8,5^{\circ} \mathrm{C}$ é uma temperatura razoavelmente baixa, o sistema catalítico em estudo mostrou-se sensível aos primeiros 10 a 20 dias de envelhecimento, apresentando uma leve desativação neste período e mudanças nas características do polímero formado. A perda de atividade, provavelmente, não é a principal responsável pelo aumento da massa molar, da viscosidade Mooney e da viscosidade em solução, pois as conversões finais dos polímeros obtidos são muito semelhantes. As características dos sítios remanescentes se modificam após o período mais crítico do envelhecimento, refletindo-se no aumento das variáveis acima e no estreitamento da polidispersão. Estas observações indicam que houve uma espécie de seleção, onde os sítios mais instáveis, geradores de polímeros de baixa massa molar.

\section{Conclusões}

O envelhecimento forçado das diferentes fases do catalisador (F1, F2, F3 e F4) afetou a conversão e a atividade catalítica de maneiras distintas. Os resultados de conversão e atividade catalítica mostraram que a fase mais afetada pelo envelhecimento forçado a $40^{\circ} \mathrm{C}$ foi a $\mathrm{F} 1$, etapa em que o versatato de neodímio é adicionado à solução de hidreto de di-isobutil-alumínio (Catalisador 447). A partir dos resultados obtidos, considerando a perda de atividade do composto formado, por exposição à temperatura, a seguinte ordem pode ser sugerida: fase da alquilação $>>$ fase da cloração $>>$ fase de envelhecimento do complexo pronto $>$ catalisador padrão. O teor de unidades 1,4-cis aumentou ligeiramente à medida que o envelhecimento forçado foi se mostrando mais efetivo com relação à influência exercida sobre as fases de preparo do catalisador. A microestrutura não se alterou com o envelhecimento natural do catalisador enquanto que a macroestrutura mostrou dependência desta variável. Foi observado um ligeiro aumento na conversão e na atividade catalítica à medida que o tempo de envelhecimento do catalisador aumentou de 1 para 10 dias.

\section{Agradecimentos}

Os autores agradecem a Petroflex Indústria e Comércio S.A. (atualmente Lanxess) pelo suporte financeiro a este trabalho.

\section{Referências Bibliográficas}

1. Friebe, L.; Nuyken, O. \& Obrecht, W. - Adv. Polym. Sci., 204, p.1 (2006). http://dx.doi.org/10.1007/12_094

2. Pires, N. M. T. - "Estudo de sistemas catalíticos à base de neodímio para a produção de polibutadieno alto-cis", Tese de Doutorado, Universidade Federal do Rio de Janeiro, Brasil (2004).

3. Mello, I. L.; Soares, B. G.; Coutinho, F. M. B. \& Nunes, D. S. S. - Polímeros, 14, p.235 (2004). http://dx.doi. org/10.1590/S0104-14282004000400008

4. Pires, N. M. T.; Coutinho, F. M. B.; Costa, M. A. S.; SantaMaria, L. C.; Mello, I. L. \& Nunes, D. S. S. - Rev. Química Industrial, 719, p.15 (2002).

5. Ferreira, C. N.; Mello, I. L. \& Coutinho, F. M. B. - Polímeros, 19, p.138 (2009). http://dx.doi.org/10.1590/ S0104-14282009000200011

6. Wilson, D. J. - J. Appl. Polym. Sci., 33, p.2505 (1995). http://dx.doi.org/10.1002/pola.1995.080331424

7. Porri, L.; Ricci, G.; Giarrusso, A.; Shubin, N. \& Lu, Z. - "Recents developments in lanthanide catalysts for 1-3-diene polymerization", in: Olefin Polymerization, cap. 2, ACS Symposium Series, v.749, P. Arjunan, J. E. 
McGrath, T. L. Hanlon (ed.), American Chemical Society, New York (1999).

8. Ricci, G.; Italia, S.; Cabassi, F. \& Porri, L. - Polym. Comunn., 28, p.223 (1987).

9. Cabassi, F.; Italia, S.; Ricci, G. \& Porri, L. "Neodymium catalysts for 1,3-diene polymerization. Some observations on their activity and stereospecificity", in: Transition Metal Catalyzed Polymerizations Ziegler-Natta and Metathesis Polymerizations, p.655, R. P. Quirk (ed.), Cambridge University Press, Cambridge (1988).

10. Oehme, A.; Gebauer, U.; Gehrke, K.; Beyer, P.; Hartmann, B. \& Lechner, M. D. - Macromol. Chem. Phy., 195, p.3773 (1994). http://dx.doi.org/10.1002/macp.1994.021951203

11. Oehme, A.; Gebauer, U.; Gehrke, K.; Beyer, P.; Hartmann, B. \& Lechner, M. D. - Angew. Makromol. Chem., 235, p.121 (1996). http://dx.doi.org/10.1002/apmc.1996.052350111

12. Mello, I. L \& Coutinho, F. M. B. - J. Appl. Polym. Sci., 112, p.1496 (2009). http://dx.doi.org/10.1002/app.29617

13. Mello, I. L.; Soares, B. G.; Coutinho, F. M. B. \& Nunes, D. S. S. - Polímeros, 17, p.62 (2007). http://dx.doi. org/10.1590/S0104-14282007000100014
14. American Society for Testing and Materials - ASTM. 164698a: standard test methods for rubber: viscosity, stress relaxation, and pre-vulcanization characteristics (Mooney Viscosity), 11 p., v.9, West Conshohocken (1999).

15. Schröder, E.; Müller, G. \& Arndt, K. F. - "Polymer characterization", Hanser Publishers, Munich (1989).

16. Kwag, G. "New ultra high cis polybutadiene technology through a novel neodymium catalyst and its vulcanizate properties", in: Proceedings of Symposium of International Rubber Conference, p.174, Beijing, China (2004).

17. Zhang, Z.; Cui, D.; Wang, B.; Liu, B. \& Yang, Y. - Struct Bond, 137, p.49 (2010). http://dx.doi. org/10.1007/430_2010_16

18. Ferreira Junior, L. C.; Nele, M.; Costa, M. A. S. \& Pinto, J. C. - Macromol. Theory Simul., 19, p.496 (2010). http:// dx.doi.org/10.1002/mats.201000039

19. Pinto, J. C.; Ferreira Junior, L. C.; Nele, M.; Costa, M. A. S.; Pires, N. M. T. \& Santa-Maria, L. C. - Polym Eng Sci., 51, p.712 (2011).

Enviado: $26 / 11 / 12$

Reenviado: $26 / 06 / 13$

Aceito: $10 / 07 / 13$ 